\title{
ОБЗОР ПРОГРАММНЫХ СРЕДСТВ МОДЕЛИРОВАНИЯ СЕТЕЙ ПЕТРИ
}

Overview of software simulation of Petri nets

Матющенко А. А. ${ }^{1}$ (Matyushchenko A.A.)

${ }^{1}$ Одессакая национальная академия пищевых технологий, г. Одесса

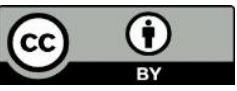

ONAFT

Open Access DOI: $10.15673 /$

\section{Аннотация}

Данная статья посвящена обзору программных продуктов, позволяющим провести моделирования сетей Петри.

\section{Abstract}

This article provides an overview of software, allowing to carry out simulation of Petri networks.

Ключевые слова

Сети Петри, моделирование, программное обеспечение.

\section{Краткие теоретические сведения о сетях Петри}

Теория сетей Петри является хорошо известным и популярным формализмом, предназначенным для работы с параллельными и асинхронными системами. Основанная в начале 60-х годов немецким математиком Карлом Адамом Петри, в настоящее время она содержит большое количество моделей, методов и средств анализа, имеющих обширное количество приложений практически во всех отраслях вычислительной техники и даже вне ее.

Сети Петри представляют собой двудольный ориентированный граф, в котором позициям соответствуют вершины, изображаемые кружками, а переходам - вершины, изображаемые прямоугольниками. Также оригинальные сети Петри были расширены такими элементами как ингибиторные (запрещающие) дуги.

Вершины одного типа не могут быть соединены. В позициях могут размещаться метки (фишки), способные перемещаться по сети через переходы. Выполнением сети Петри управляют количество и распределение фишек сети. Фишки находятся в кружках и управляют выполнением переходов сети.

Сеть Петри выполняется посредством запусков переходов. Запуск перехода может быть осуществлен только в случае, когда во всех входных позициях присутствует хотя бы одна фишка. Одна фишка отнимается из каждой входной позиции и добавляется по одной во все выходные. Единовременно может сработать только один переход, что решает проблему конфликтов (когда одна позиция связанна с несколькими переходами готовыми к выполнению).

Для удобства, чтобы не перерисовывать каждый раз всю сеть, необходимо использовать программное обеспечение, позволяющее производить интерактивное моделирование. В данной статье хотелось бы показать доступные на данный момент программные среды моделирования.

\section{PIPE2}

Platform Independent Petri net Editor (платформо-независимый редактор сетей Петри) - представляет разработку, начатую в 2003 году, группой аспирантов отделения компьютерных наук Имперского колледжа. Лондон. Разработка ведется по принципу открытого программного обеспечения, что позволяет любому желающему внести свой вклад в реализацию.

PIPE2 предоставляет возможность редактировать и моделировать стохастические сети Петри с запрещающими дугами. Также имеется возможность просматривать матрицы инцидентности для текущей маркировки, а в режиме моделирования создается список сработавших переходов и отражение изменения количества маркеров в позициях. Для каждой дуги можно задать весовой коэффициент. 


\section{СТУДЕНТСЬКА НАУКА}

STUDENT SCIENCE

Благодаря использованию кроссплатформенного языка программирования JAVA программу можно запускать на различных операционных системах, что позволяет использовать PIPE2 на привычной для исследователя платформе.

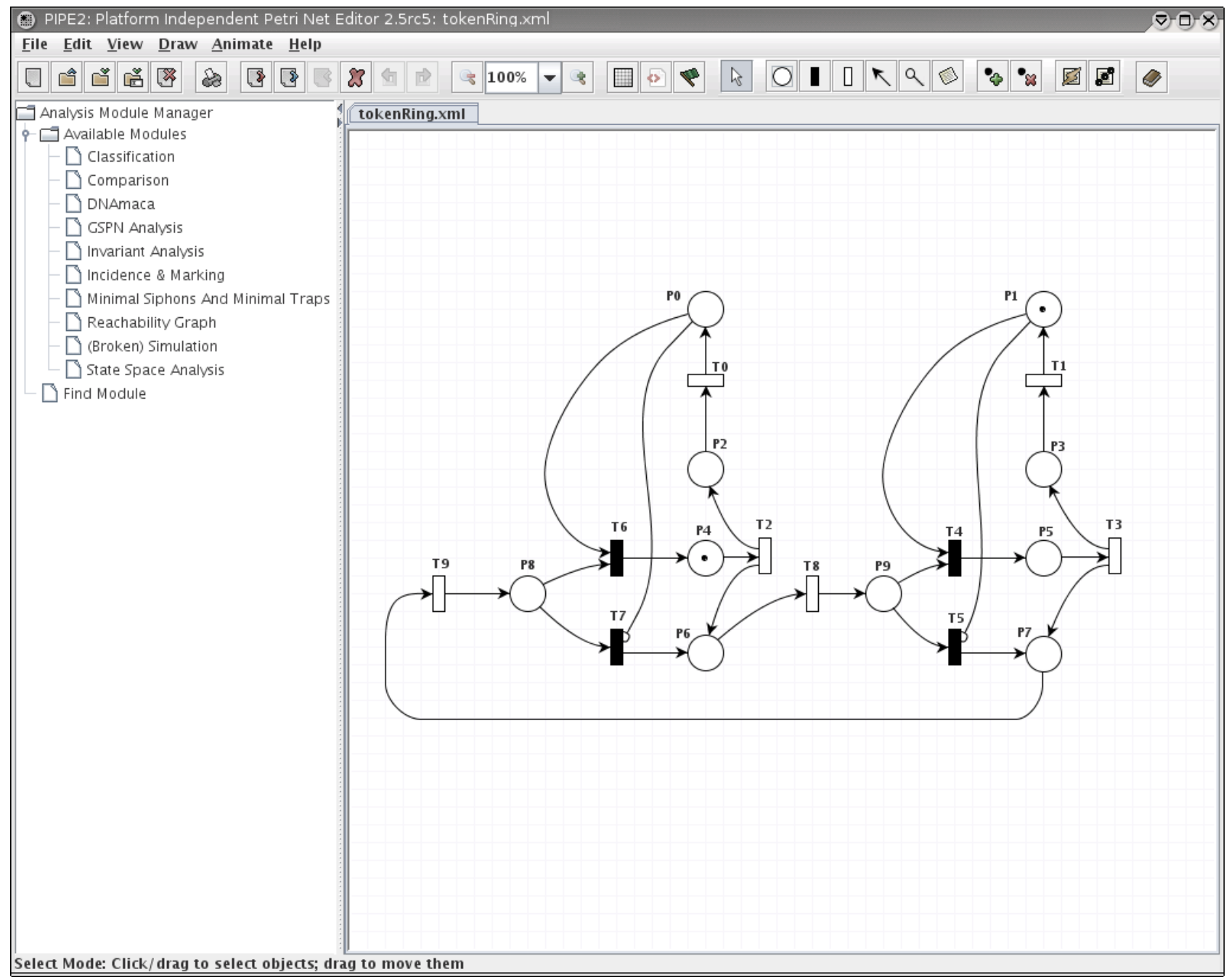

Рис. 1 - Вид главного окна программы с набранной сетью Петри.

Petri.Net Simulator 2

Petri.Net Simulator 2 - приложение, созданное для рисования, моделирование и симуляции гибких производственных систем при помощи аппарата сетей Петри, но могут быть использованы для других дискретных событийных систем.

Приемущество данного симулятора заключается что настройки и все функции распределены по своим прикрепляимым окнам. А также имеют понятную структуру и общий вид. 


\section{СТУДЕНТСЬКА НАУКА STUDENT SCIENCE}

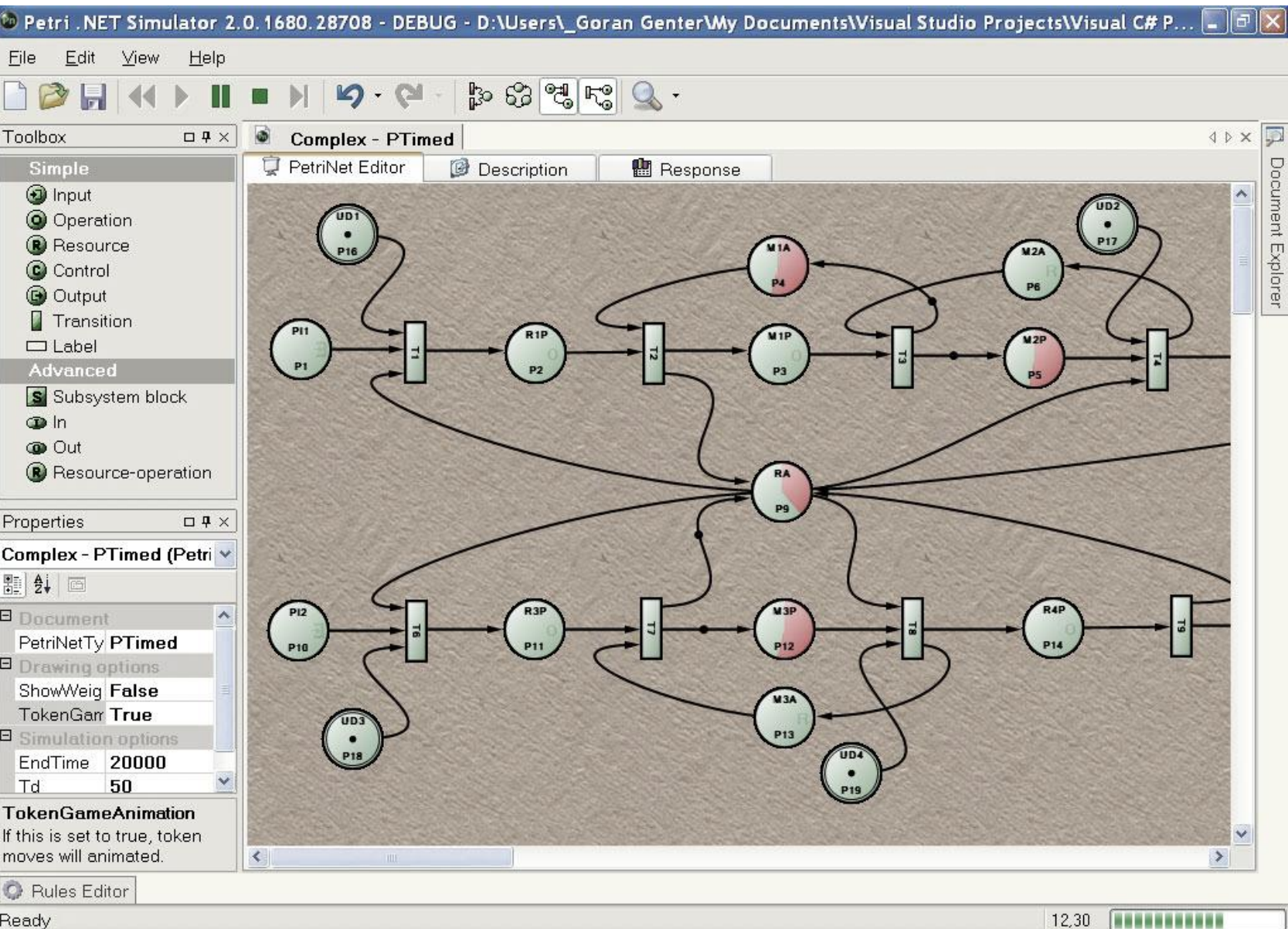

Рис. 2. Вид главного окна Petri.Net Simulator 2 в процессе моделирования.

Реализовано множество дополнительных видов позиций, которые позволяют полностью использовать гибкость математического аппарата сетей Петри для описания дискретно событийных систем.

Например есть возможность временной задержки срабатывания позиции, что позволяет реализовать более сложные алгоритмы передвижения маркеров по сети. Также можно вводить свои правила для срабатывания позиции, это реализовывается через специальное окно Rules Editor. Где при помощи простого синтаксиса можно на основании логического условия помещать маркеры в определенные позиции, без отбора маркеров из позиций участвующих в условии.

Для каждого перехода можно задать весовой коэффициент, а также приоритет что позволяет избежать коллизий, если это позволяет алгоритм функционирования моделируемой сети. При не указании или при равных значениях приоритета, переход ведет себя как в обычной стохастической сети Петри.

Реализована возможность задания ингибиторной дуги на определенные типы переходов. Также есть функция объединения нескольких элементов в группу, так называемый «черный ящик», есть только входы и выходы, а внутреннюю

\section{DCnet}

DCNET - имеет широкие возможности по моделированию сетей Петри, а также построенное на их основании дискретно-непрерывных систем с управляемой структурой, вот что следует из описания программы DCNET предназначена:

- для моделирования и исследования гибких автоматизированных производств, АСУТП совместно с технологическим объектом управления, автономно функционирующих объектов космического, воздушного, наземного, водного и подводного назначения

• д для проектирования электронных экзаменаторов, динамических и пультовых тренажеров

- для разработки экспертных систем. 


\section{СТУДЕНТСЬКА НАУКА STUDENT SCIENCE}

Основными режимами работы программы являются:

- Редактирование;

- редактирование маркировки сети;

- моделирование.

Режим редактирования предназначен для графического ввода топологической структуры, элементов и параметров дискретно-непрерывной сети СУС и позволяет: оперативно получать информацию о структуре схемы и параметров ее элементов; добавлять и удалять элементы, изменять их параметры, число входов и выходов, а также соединения между ними; осуществлять копирование и перемещение отдельных блоков, их групп, как с сохранением связей, так и без, что ускоряет набор схемы; строить иерархические модели СУС из библиотеки готовых подмоделей/

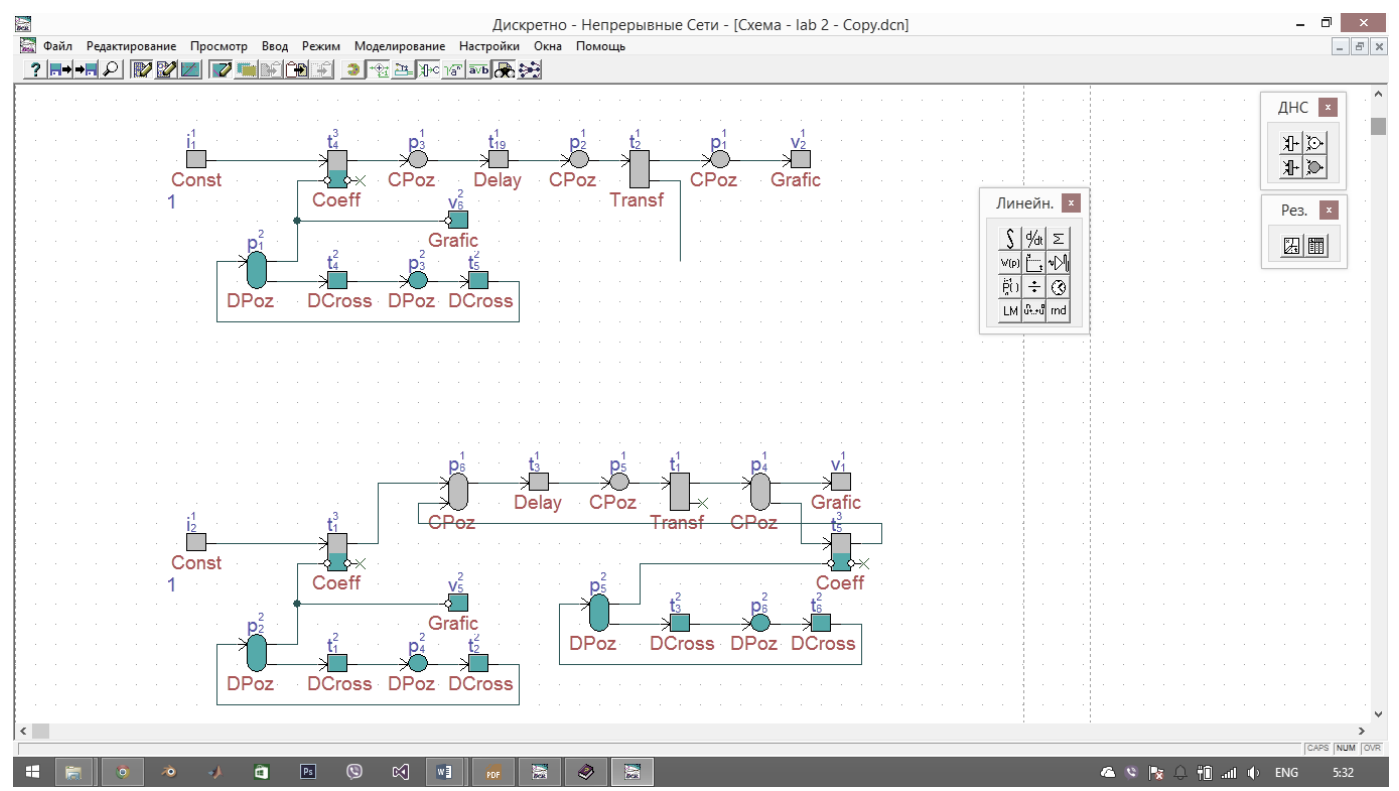

Рис. 3.Вид главного окна DCnet с введенной сетью Петри.

Вывод

Данная показывает текущее положение в сфере моделирования математического аппарата сетей Петри на сегодняшний день. Единственный не заброшенным проектом, который активно развивается является РІРЕ2, самым удобным в плане использования оказался Petri.net simulator, а программный продукт с самым функциональным математическим ядром является DCnet. Как видно у каждой из программ есть свои достоинства, и это несомненно радует.

\section{Литература}

1. Описание продукта pipe2 на официальном сайте/ http://pipe2.sourceforge.net/about.html;

2. Статья Википедии о сетях Петри/ http://ru.wikipedia.org/wiki/Сети_петри;

3. Теория сетей Петри/ http://www.iacp.dvo.ru/lab 11/otchet/ot2000/pn3.html;

4. Описание программы Petri.Net / http://goo.gl/jx11Qi;

5. Программная среда моделирования систем с управляемой структурой / В.А. Денисенко, А.Г. Нестерюк, B.В. Ляхин// http://storage.library.opu.ua/online/periodic/opu_1998_2(6)/1_3.htm

\section{References}

1. Opisanie produkta pipe2 na ofitsialnom sayte/ http://pipe2.sourceforge.net/about.html;

2. Statya Vikipedii o setyah Petri/ http://ru.wikipedia.org/wiki/Seti_petri;

3. Teoriya setey Petri/ http://www.iacp.dvo.ru/lab_11/otchet/ot2000/pn3.htm;

4. Opisanie programmyi Petri.Net/ http://goo.gl/jx11Qi;

5. Programmnaya sreda modelirovaniya sistem s upravlyaemoy strukturoy / V.A. Denisenko, A.G. Nesteryuk, V.V. Lyahin. 